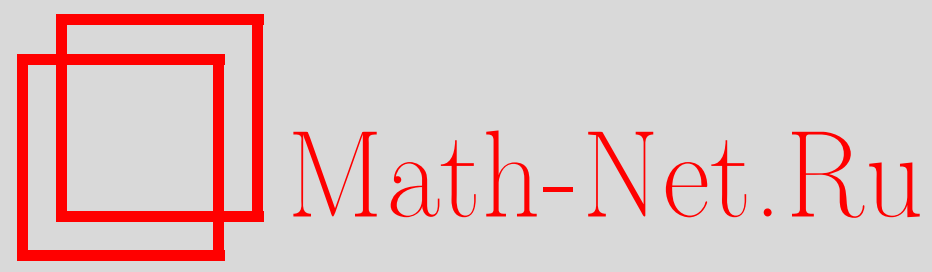

А. А. Боровков, Об асимптотике распределений времен первого прохождения, I, Матем. заметки, 2004, том 75, выпуск 1, 24-39

DOI: https://doi.org/10.4213/mzm4

Использование Общероссийского математического портала Math-Net.Ru подразумевает, что вы прочитали и согласны с пользовательским соглашением http://www.mathnet.ru/rus/agreement

Параметры загрузки:

IP : 18.234 .197 .8

26 апреля 2023 г., 09:46:48 
УДК 519.214

\section{ОБ АСИМПТОТИКЕ РАСПРЕДЕЛЕНИЙ ВРЕМЕН ПЕРВОГО ПРОХОЖДЕНИЯ, І}

\section{А. А. Боровков}

В работе найдена асимптотика и получены оценки для распределения времен первого прохождения случайным блужданием фиксированного и растущего уровней. В первой части работы рассмотрен случай нулевого уровня.

Библиография: 24 названия.

\section{Введение}

Пусть $\xi, \xi_{1}, \xi_{2}, \ldots$ - независимые одинаково распределенные случайные величины, $S_{n}=\sum_{j=1}^{n} \xi_{j}$,

$$
\eta_{+}(x)=\min \left\{k \geqslant 1: S_{k}>x\right\}, \quad \eta_{-}(x)=\min \left\{k \geqslant 1: S_{k} \leqslant-x\right\}, \quad x \geqslant 0,
$$

где мы полагаем $\eta_{+}(x)=\infty$ (соответственно $\left.\eta_{-}(x)=\infty\right)$, если все $S_{k} \leqslant x$ (соответственно $\left.S_{k}>-x\right), k=1,2, \ldots$.

Целью настоящей работы является отыскание и оценка асимптотик вероятностей $\mathrm{P}\left(\eta_{-}(x)=n\right)$ и $\mathrm{P}\left(\eta_{+}(x)=n\right)$ или их интегральных аналогов $\mathrm{P}\left(n<\eta_{-}(x)<\infty\right)$ и $\mathrm{P}\left(n<\eta_{+}(x)<\infty\right)$ при $n \rightarrow \infty$. Уровень $x \geqslant 0$ может быть как фиксированным, так и растушим вместе с $n$. Особое внимание будет уделено случайньм величинам $\eta_{ \pm}=\eta_{ \pm}(0)$.

Определяющую роль при изучении названных асимптотик играет "снос" блуждания, который мы будем характеризовать с помощью значений

$$
D=D_{+}=\sum_{k=1}^{\infty} \frac{\mathrm{P}\left(S_{k}>0\right)}{k} \quad \text { и } \quad D_{-}=\sum_{k=1}^{\infty} \frac{\mathrm{P}\left(S_{k} \leqslant 0\right)}{k}
$$

где, очевидно, $D_{+}+D_{-}=\infty$.

Хорошо известно, что (см., например, [1], [2])

$$
\begin{aligned}
\left\{D_{+}<\infty, D_{-}=\infty\right\} & \Leftrightarrow\left\{\eta_{-}<\infty \text { п.н., } p=\mathrm{P}\left(\eta_{+}=\infty\right)>0\right\} \\
& \Leftrightarrow\{\underline{S}=-\infty, \bar{S}<\infty \text { п.н. }\}, \\
\left\{D_{+}=\infty, D_{-}=\infty\right\} & \Leftrightarrow\left\{\eta_{-}<\infty, \eta_{+}<\infty \text { п.н. }\right\} \Leftrightarrow\{\underline{S}=-\infty, \bar{S}=\infty \text { п.н. }\},
\end{aligned}
$$

Работа выполнена при частичной финансовой поддержке грантами Российского фонда фундаментальных исследований, гранты №№ 02-01-00902, № 00-15-96178, и фонда INTAS, проект № 00-265. 
где

$$
\bar{S}=\max _{k \geqslant 0} S_{k}, \quad \underline{S}=\min _{k \geqslant 0} S_{k}
$$

Очевидно, что справедливо также соотношение, “симметричное” к (1) для события $\left\{D_{-}<\infty, D_{+}=\infty\right\}$. Учитывая эту симметрию, можно ограничиться рассмотрением случая

$$
A_{0}=\left\{D_{-}=\infty, D_{+}=\infty\right\}
$$

и лишь одной из двух возможностей

$$
A_{-}=\left\{D_{-}=\infty, D_{+}<\infty\right\} \quad \text { или } \quad A_{+}=\left\{D_{-}<\infty, D_{+}=\infty\right\} .
$$

Если $\mathrm{E} \xi=a$ существует, то

$$
A_{0}=\{a=0\}, \quad A_{-}=\{a<0\}, \quad A_{+}=\{a>0\}
$$

В дальнейшем классификация результатов будет происходить по трем основным признакам:

1) по значению $x$ (мы будем различать случаи $x=0, x>0$ фиксированно и $x \rightarrow$ $\infty)$

2) по направлению сноса (одна из возможностей $A_{0}, A_{ \pm}$);

3) по характеру распределения $\xi$.

В соответствии с этим работа имеет следующую структуру. Она разбита на три раздела (главньм образом в соответствии с тремя упомянутыми возможностями для $x$ ). Последние два раздела входят во вторую часть заметки (публикуемую отдельно).

В разделе 1 изучается случай $x=0, A_{-}$. Пункты 1.1-1.3 посвящены различным классам хвостов распределения $\xi$.

В разделе 2 рассмотрен случай фиксированного $x \geqslant 0$ при выполнении условий $A_{0}$, $A_{-}$. Там рассмотрена, в частности, связь распределений $\eta_{ \pm}(x)$ и $\eta_{ \pm}$при фиксированном $x>0$.

В разделе 3 изучается случай, когда $x \rightarrow \infty$ вместе с $n$. В пш. $3.1-3.3$ рассмотрены различные классы хвостов распределения $\xi$.

\section{1. Случай $x=0, A_{-}$}

1.1. Вводная часть. Здесь мы будем изучать главньм образом асимптотику

$$
\mathrm{P}\left(\eta_{-}>n\right) \quad \text { и } \quad \mathrm{P}\left(\eta_{+}=n\right)
$$

а также тесно связанную с ними асимптотику $\mathrm{P}(\theta=n)$ для величины

$$
\theta=\min \left\{n: S_{n}=\bar{S}\right\}
$$

времени первого достижения максимума $\bar{S}$. Эта связь имеет простой вид:

$$
\mathrm{P}(\theta=n)=\mathrm{P}\left(\bar{S}_{n-1}<S_{n}=\bar{S}_{n}\right) \mathrm{P}\left(\max _{k \geqslant n}\left(S_{k}-S_{n}\right)=0\right),
$$


(первьй множитель в правой части равен 1 при $n=0)$, где $\bar{S}_{n}=\max _{k} \leqslant n S_{k}$,

$$
\mathrm{P}\left(\bar{S}_{n-1}<\bar{S}_{n}=S_{n}\right)=\mathrm{P}\left(\underline{S}_{n}^{1}>\underline{S}_{n}=0\right),
$$

$\underline{S}_{n}^{1}=\min _{1 \leqslant k \leqslant n} S_{k}, \underline{S}_{n} \equiv \min _{0 \leqslant k \leqslant n} S_{k}$, так что

$$
\mathrm{P}(\theta=n)=p \mathrm{P}\left(\underline{S}_{n}^{1}>\underline{S}_{n}=0\right)=p \mathrm{P}\left(\eta_{-}>n\right)
$$

при $p=\mathrm{P}(\bar{S}=0)=\mathrm{P}\left(\eta_{+}=\infty\right)=1 / \mathrm{E} \eta_{-}$(по поводу последних равенств см. ниже теорему 1 или [1]-[3]).

Мы будем рассматривать следующие классы распределений и их расширения:

$\mathscr{R}$ - класс регулярных распределений, когда $\mathrm{P}(\xi>x)=V(x)$, где

$$
V(x)=x^{-\alpha} L(x), \quad \alpha>1
$$

$L(x)$ - медленно меняющаяся функция (ММ $\Phi)$.

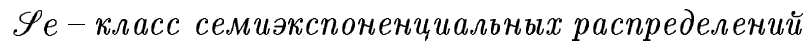

$$
\mathrm{P}(\xi>x)=V(x)=e^{-l(x)}, \quad l(x)=x^{\alpha} L(x), \quad \alpha \in(0,1),
$$

где $L-\mathrm{MM} \Phi$ такая, что при $\Delta=o(x), x \rightarrow \infty$, и любом $\varepsilon>0$

$$
\begin{aligned}
& l(x+\Delta)-l(x) \sim \frac{\alpha \Delta l(x)}{x}, \quad \text { если } \frac{\alpha \Delta l(x)}{x}>\varepsilon \\
& l(x+\Delta)-l(x) \rightarrow 0, \quad \text { если } \frac{\alpha \Delta l(x)}{x} \rightarrow 0 .
\end{aligned}
$$

Для вьполнения (6) достаточно, чтобы $L$ была дифференцируема "на бесконечности", $L^{\prime}(x)=o(L(x) / x)$. Как видно из определения, к классу $\mathscr{S} e$ можно относить и все функции $V(x)=e^{-\tilde{l}(x)}$, где $\tilde{l}(x)=l(x)+o(1)$ при $x \rightarrow \infty, l$ удовлетворяет $(6)$, так что функция $L$ для $V \in \mathscr{S}$ е не обязательно должна быть дифференцируемой.

Распределения из классов $\mathscr{R}$ и $\mathscr{S}$ е являются субәкспоненииальными, т.е. обладают свойствами

$$
V^{*(2)}(x) \sim 2 V(x), \quad \frac{V(x+v)}{V(x)} \rightarrow 1
$$

при $x \rightarrow \infty$ и любом фиксированном $v$, где $V^{*(2)}$ - свертка “хвоста” $V$ с собой:

$$
V^{*(2)}(x)=\int_{-\infty}^{\infty} \mathrm{P}(\xi \in d t) V(x-t)=-\int_{-\infty}^{\infty} V(x-t) d V(t)
$$

Те свойства хвостов $V \in \mathscr{R}$ и $V \in \mathscr{S} e$, которые будут нужны нам ниже, сохранятся, если классы $\mathscr{R}$ и $\mathscr{S}$ е несколько расширить, разрешив функциям $V$ "медленно колебаться" (см. ниже). Такие расширения также будут рассмотрены.

Альтернативньм к $\mathscr{R}$ и $\mathscr{S}$ е является класс $\mathscr{C}$ распределений, убывающих әкспоненииально быстро, т.е. распределений, для которых выполнено условие Крамера:

$$
\mu=: \sup \{\lambda: \varphi(\lambda)<\infty\}>0, \quad \text { где } \varphi(\lambda)=: \mathrm{E} e^{\lambda \xi} \text {. }
$$


Здесь мы будем различать две возможности:

$$
\begin{array}{ll}
\text { 1) } \lambda_{0} \leqslant \mu, & \varphi^{\prime}\left(\lambda_{0}\right)=0, \\
\text { 2) } \lambda_{0}=\mu, \quad \varphi^{\prime}(\mu) & <0,
\end{array}
$$

где $\lambda_{0}-$ точка, в которой достигается $\min \varphi(\lambda):=\varphi$. Очевидно, что в первом случае всегда $\varphi^{\prime}\left(\lambda_{0}\right)=0$, если $\lambda_{0}<\mu$.

Обратимся теперь к предшествуюшим результатам. Буквой $c$ с индексами или без мы будем обозначать постоянные, не всегда одни и те же, если они появляются в разных формулах.

Полный асимптотический анализ $\mathrm{P}\left(\eta_{ \pm}(x)=n\right)$ при всех $x$ и $n \rightarrow \infty$ для ограниченных решетчатых $\xi$ проведен в [4].

Для некоторого расширения класса $\mathscr{R}$ регулярных распределений асимптотика $\mathrm{P}(\theta=n) \sim c V(-a n)$, a, стало быть, и асимптотика $\mathrm{P}\left(\eta_{-}>n\right)$ была найдена в [3]. $\mathrm{B}$ той же монографии было установлено, что для класса $\mathscr{C}, \lambda_{0}<\mu$,

$$
\mathrm{P}(\theta=n) \sim \frac{c \varphi^{n}}{n^{3 / 2}} \quad\left(\sim p \mathrm{P}\left(\eta_{-}>n\right)\right)
$$

Весьма полные результаты об асимптотике $\mathrm{P}\left(\eta_{-}(x)>n\right)$ и $\mathrm{P}\left(n<\eta_{+}(x)<\infty\right)$ при фиксированном $x \geqslant 0$ и для классов $\mathscr{R}$ и $\mathscr{C}$ получены в [5]-[9]. В связи со сказанным следует отметить, что часть результатов теорем $2,5,6,8$ является известной. Тем не менее, мы приводим их здесь для полноты и систематичности изложения.

Необходимые и достаточные условия для конечности $\mathrm{E}\left(\eta_{-}\right)^{\gamma}, \gamma \geqslant 1$, и близкие задачи изучены в [10]-[13]. Установлено, в частности, что $\mathrm{E} \eta_{-}^{\gamma}<\infty, \gamma>0$, тогда и только тогда, когда $\mathrm{E}\left(\xi^{+}\right)^{\gamma}<\infty$, где $\xi^{+}=\max (0, \xi)$ (см., например, [10]). Неулучшаемые оценки для $\mathrm{P}\left(\eta_{-}>n\right)$ приведены в $[14, \S 43]$.

Так как связь (3) распределений $\theta$ и $\eta_{-}$установлена, то в дальнейшем мы ограничимся рассмотрением лишь распределений $\eta_{ \pm}$.

Мы начнем с соотношений, проясняющих связь распределений $\eta_{-}$и $\eta_{+}$, которая представляет самостоятельньй интерес. Введем в рассмотрение случайную величину $\zeta$ с производящей функцией

$$
H(z)=: \mathrm{E} z^{\zeta}=\frac{1-\mathrm{E} z^{\eta_{-}}}{(1-z) \mathrm{E} \eta_{-}}
$$

так что

$$
\mathrm{P}(\zeta=k)=\frac{\mathrm{P}\left(\eta_{-}>k\right)}{\mathrm{E} \eta_{-}}, \quad k=0,1, \ldots,
$$

и обозначим $p(z)=\mathrm{E}\left(z^{\eta_{+}} / \eta_{+}<\infty\right), p=\mathrm{P}\left(\eta_{+}=\infty\right)=\mathrm{P}(\bar{S}=0), q=1-p=$ $\mathrm{P}\left(\eta_{+}<\infty\right)$.

ТЕОРема 1. Справедливы следующие соотношения:

1) функиия

$$
H(z)=\frac{1-q}{1-q p(z)}
$$

u, стало быть, распределение $\eta_{+}$полностью определяет распределение $\eta_{-}$и наоборот; 
2) при всех $n=1,2, \ldots$

$$
\mathrm{P}\left(\eta_{+}=n\right)<\mathrm{P}\left(\eta_{-}>n\right)
$$

3) если последовательность $\mathrm{P}\left(\eta_{+}=k\right) / q$ субәкспоненииальна, то при $n \rightarrow \infty$

$$
\mathrm{P}\left(\eta_{-}>n\right) \sim \frac{\mathrm{P}\left(\eta_{+}=n\right)}{p^{2}}, \quad p=\frac{1}{\mathrm{E} \eta_{-}}
$$

если последовательность

$$
b_{n}=\frac{\mathrm{P}\left(S_{n}>0\right)}{n D}
$$

$2 \partial e$

$$
D=\sum_{k=1}^{\infty} \frac{\mathrm{P}\left(S_{k}>0\right)}{k}=-\ln p
$$

субэкспоненциальна, то

$$
\mathrm{P}\left(\eta_{-}>n\right) \sim e^{D} \frac{\mathrm{P}\left(S_{n}>0\right)}{n}, \quad \mathrm{P}\left(\eta_{+}=n\right) \sim e^{-D} \frac{\mathrm{P}\left(S_{n}>0\right)}{n}
$$

4) случайная величина $\zeta$ имеет безгранично делимое распределение и допускает представление

$$
\zeta=\omega_{1}+\cdots+\omega_{\nu}
$$

әде $\omega_{1}, \omega_{2}, \ldots$ - независимые копии случайной величины $\omega$,

$$
\begin{gathered}
\mathrm{P}(\omega=k)=\frac{\mathrm{P}\left(S_{k}>0\right)}{k D} ; \\
D=\sum \frac{\mathrm{P}\left(S_{k}>0\right)}{k}=-\ln p, \quad p=\frac{1}{\mathrm{E} \eta_{-}}
\end{gathered}
$$

$\nu$ не зависит от $\left\{\omega_{i}\right\}$ и имеет распределение Пуассона с параметром $D ;$ из (15) вытекает, что

$$
\mathrm{P}\left(\eta_{-}>n\right)=\mathrm{E} \eta_{-} \mathrm{P}\left(\omega_{1}+\cdots+\omega_{\nu}=n\right)
$$

ЗАмЕчАниЕ 1. Утверждение теоремы о том, что распределения $\eta_{ \pm}$определяют друг друга, выглядит несколько неожиданно, поскольку аналогичное утверждение для величин первой положительной и первой неположительной сумм $\chi_{+}=S_{\eta_{+}}$и $\chi_{-}=S_{\eta_{-}}$не верно. Действительно, если

$$
\mathrm{P}(\xi \leqslant-x)=c e^{-h x} \quad \text { при } \quad x \geqslant 0, \quad c<1, \quad h>0,
$$

то при любом распределении $\xi$ на положительной полуоси $(0, \infty)$ будет справедливо $\mathrm{P}\left(\chi_{-}<-x\right)=e^{-h x}$, в то время как

$$
1-\mathrm{E}\left(e^{i \lambda \chi_{+}} ; \eta_{+}<\infty\right)=\frac{(1-\varphi(i \lambda))(i \lambda+h)}{i \lambda}
$$

и, стало быть, распределения $\mathrm{P}\left(\chi_{+}>x ; \eta_{+}<\infty\right)$ будут разньпи для разньх распределений $\mathrm{P}(\xi>x)$ при $x>0$. 
ДокАЗАТЕЛЬСтво тЕоремЫ 1. Доказательство теоремы 1 (а также теоремы 2 ниже) будет следовать в основном по пути, по которому шло изучение асимптотики $\mathrm{P}(\theta=$ $n)$ в $[3, \S 21]$. В его основе лежат факторизационные тождества

$$
\begin{gathered}
1-\mathrm{E} z^{\eta_{-}}=\exp \left\{-\sum_{k=1}^{\infty} \frac{z^{k}}{k} \mathrm{P}\left(S_{k} \leqslant 0\right)\right\}, \\
1-\mathrm{E}\left(z^{\eta_{+}} ; \eta_{+}<\infty\right)=\exp \left\{-\sum_{k=1}^{\infty} \frac{z^{k}}{k} \mathrm{P}\left(S_{k}>0\right)\right\}, \quad|z| \leqslant 1
\end{gathered}
$$

(см., например, [1]-[3]). Поскольку

$$
(1-z)=e^{\ln (1-z)}=\exp \left\{-\sum_{k=1}^{\infty} \frac{z^{k}}{k}\right\}
$$

тождества (18), (19) можно записать в виде

$$
\begin{gathered}
\frac{1-\mathrm{E} z^{\eta_{-}}}{1-z}=\sum_{k=0}^{\infty} z^{k} \mathrm{P}\left(\eta_{-}>n\right)=\exp \left\{\sum_{k=1}^{\infty} \frac{z^{k}}{k} \mathrm{P}\left(S_{k}>0\right)\right\}, \\
\sum_{k=0}^{\infty} z^{k} \mathrm{P}\left(\eta_{+}=n\right)=1-\exp \left\{-\sum_{k=0}^{\infty} \frac{z^{k}}{k} \mathrm{P}\left(S_{k}>0\right)\right\} .
\end{gathered}
$$

Полагая здесь $z=1$, получим соотношения $D=-\ln p, p=1 / \mathrm{E} \eta_{-}$.

Утверждение (10) вытекает из сравнения (20) и (21). Неравенство (11) немедленно следует из (10).

Докажем теперь третье утверждение теоремы. Введем в рассмотрение целые функции

$$
\mathscr{A}_{-}(v)=e^{v D} \quad \text { и } \quad \mathscr{A}_{+}(v)=1-e^{-v D} .
$$

Тогда соотношения $(20),(21)$ при $|z| \leqslant 1$ можно записать в виде

$$
\begin{gathered}
\sum_{n=0}^{\infty} z^{n} \mathrm{P}\left(\eta_{-}>n\right)=\mathscr{A}_{-}(b(z)), \\
\sum_{n=1}^{\infty} z^{n} \mathrm{P}\left(\eta_{+}=n\right)=\mathscr{A}_{+}(b(z)), \\
b(z)=\frac{1}{D} \sum_{n=1}^{\infty} \frac{z^{n} \mathrm{P}\left(S_{n}>0\right)}{n} \equiv \sum_{n=1}^{\infty} z^{n} b_{n}
\end{gathered}
$$

где $b_{n}$ определены в (13). Так как по условию последовательность $b_{n}$ субэкспоненциальна, то остается воспользоваться известньми теоремами (см., например, [15]) о функциях от распределений (определяемых функциями $\mathscr{A}_{ \pm}$в $\left.(22),(23)\right)$.

Так как функции $\mathscr{A}_{ \pm}$целые, $\mathscr{A}_{-}^{\prime}(1)=D e^{D}, \mathscr{A}_{+}^{\prime}(1)=D e^{-D}$, то в силу $(22),(23)$ и результатов [15]

$$
\mathrm{P}\left(\eta_{-}>n\right) \sim b_{n} \mathscr{A}_{-}^{\prime}(1)=D e^{D} b_{n}, \quad \mathrm{P}\left(\eta_{+}=n\right) \sim b_{n} \mathscr{A}_{+}^{\prime}(1)=D e^{-D} b_{n} .
$$


Это доказьвает (14). Соотношение (12) доказьвается совершенно аналогично, учитывая тот факт, что функция $\mathscr{A}(v)=(1-q) /(1-q v)$ аналитична в области $|v|<1 / q$, $\mathscr{A}^{\prime}(1)=q /(1-q)$ и в силу $(10) H(z)=\mathscr{A}(p(z))$. Поэтому

$$
\frac{\mathrm{P}\left(\eta_{-}>n\right)}{\mathrm{E} \eta_{-}} \sim \frac{q}{1-q} \frac{\mathrm{P}\left(\eta_{+}=n\right)}{q}
$$

что равносильно (12).

Утверждение (15) получается, если заметить, что

$$
H(z)=\exp \left\{\sum_{k=1}^{\infty} \frac{z^{k} \mathrm{P}\left(S_{k}>0\right)}{k}-D\right\}=\exp \{D(Q(z)-1)\}
$$

где

$$
Q(z)=\sum_{k=1}^{\infty} \frac{z^{k} \mathrm{P}\left(S_{k}>0\right)}{k D}
$$

Ясно, что правая часть в $(24)$ есть производящая функция $\omega_{1}+\cdots+\omega_{\nu}$. Теорема доказана.

Перейдем теперь к “явным” асимптотическим представлениям для распределений $\eta_{ \pm}$ в терминах распределения $\xi$.

\section{2. Случай $a=\mathrm{E} \xi<0, x=0$, классы $\mathscr{R}$ и $\mathscr{S} е$ и их расширения.}

ТЕорема 2. Пусть распределение $\xi$ принадлежит одному из классов $\mathscr{R}$ или $\mathscr{S}$ e, при этом в случае $V \in \mathscr{S}$ е предполагается, что $\alpha<1 / 2$. Тогда при $n \rightarrow \infty$

$$
\begin{aligned}
& \mathrm{P}\left(\eta_{-}>n\right) \sim V(|a| n) e^{D}, \\
& \mathrm{P}\left(\eta_{+}=n\right) \sim V(|a| n) e^{-D},
\end{aligned}
$$

əде D определено в (16),

$$
e^{D}=\mathrm{E} \eta_{-}, \quad e^{-D}=\mathrm{P}(\bar{S}=0)=\mathrm{P}\left(\eta_{+}=\infty\right)
$$

Точно такое же утверждение справедливо для “дуальной” пары случайных величин

$$
\eta_{+}^{0}=\min \left\{k \geqslant 1: S_{k} \geqslant 0\right\}, \quad \eta_{-}^{0}=\min \left\{k: S_{k}<0\right\}
$$

ТЕОрема 2А. Пусть выполнены условия теоремы 2. Тогда при $n \rightarrow \infty$

$$
\mathrm{P}\left(\eta_{-}^{0}>n\right) \sim V(|a| n) e^{D^{0}}, \quad \mathrm{P}\left(\eta_{+}^{0}=n\right) \sim V(|a| n) e^{-D^{0}}
$$

əde

$$
D^{0}=\sum_{k=1}^{\infty} \frac{\mathrm{P}\left(S_{k} \geqslant 0\right)}{k}, \quad e^{D^{0}}=\mathrm{E} \eta_{-}^{0}=\frac{1}{\mathrm{P}\left(\eta_{+}^{0}=\infty\right)}
$$


ЗАмечАниЕ 2. Оба утверждения теоремы 2 допускают простую “физическую” интерпретацию. Заметим предварительно, что в условиях теоремы вероятность того, что за время $m$ произойдет большой скачок величиной $x \sim c n$ и более, равна $\approx m V(x)$. Далее, редкое событие $\left\{\eta_{-}>n\right\}$ ( $n$ велико) происходит, грубо говоря, тогда, когда в течение времени $\eta_{-}$(со средним $\mathrm{E} \eta_{-}$) произойдет большой скачок величиной $|a| n$ (после чего потребуется в среднем время $n$, чтобы достичь отрицательной полуоси). Поэтому естественно ожидать, что $\mathrm{P}\left(\eta_{-}>n\right) \sim \mathrm{E} \eta_{-} V(|a| n)$.

Аналогичную интерпретацию допускают результаты [16] о том, что $\mathrm{P}\left(\bar{S}_{\eta_{-}}>x\right) \sim$ $V(x) \mathrm{E} \eta-$.

Редкое событие $\left\{\eta_{+}=n\right\}$ происходит тогда, когда сначала (до момента $n$ ) траектория не покидает отрицательную полуось (вероятность этого близка к $\mathrm{P}(\bar{S}=0)$; в момент $n$ траектория будет "в окрестности" точки $a n)$, а затем в момент $n$ произойдет большой скачок величиной $\geqslant-a n$. Эти соображения объясняют в какой-то мере (26).

ДоКАЗАТЕЛЬСТво ТЕОРЕмЫ 2. Теорема 2 является простым следствием третьего утверждения теоремы 1 (см. (13), (14)). Нам надо найти асимптотику $b_{n}$ и убедиться, что эта последовательность субэкспоненциальна.

Вероятность $\mathrm{P}\left(S_{n}>0\right)$ можно записать в виде $\mathrm{P}\left(S_{n}^{0}>|a| n\right)$, где $S_{k}^{0}=S_{k}-a k$, $\mathrm{E} S_{k}^{0}=0$. Известно, что если $V \in \mathscr{R}$, то

$$
\mathrm{P}\left(S_{n}^{0}>|a| n\right) \sim n V(|a| n)
$$

(см., например, [17], [18]). То же соотношение справедливо, если $V \in \mathscr{S} e$ (вьполнено (5), (6)) и $\alpha<1 / 2$ (см., например, [18], [19]; при $\alpha \geqslant 1 / 2$ уклонения $x=|a| n$ не попадают в область, где справедливо (28)). Таким образом, при вьполнении условий теоремы 2

$$
b_{n} \sim \frac{V(|a| n)}{D}
$$

и последовательность $b_{n}$ субэкспоненциальна, т.е. $\sum_{k=0}^{n} b_{k} b_{n-k} \sim 2 b_{n}$ при $n \rightarrow \infty$. Теорема доказана.

Из теоремы вытекает, что при $n \rightarrow \infty$ выполнено (12), т.е.

$$
\mathrm{P}\left(\eta_{+}=n\right) \sim p^{2} \mathrm{P}\left(\eta_{-}>n\right),
$$

и что для любой возрастающей функции $f$ при $F(t)=\int_{0}^{t} f(u) d u$ справедливо

$$
\begin{aligned}
\left\{\mathrm{E} f\left(\eta_{-}\right)<\infty\right\} & \left.\mathrm{E}\left(f\left(\frac{\xi}{|a|}\right) ; \xi>0\right)<\infty\right\}, \\
\left\{\mathrm{E}\left(f\left(\eta_{+}\right) ; \eta_{+}<\infty\right)<\infty\right\} & \Longleftrightarrow\left\{\mathrm{E}\left(F\left(\frac{\xi}{|a|}\right) ; \xi>0\right)<\infty\right\} .
\end{aligned}
$$

Теорема 2А доказывается точно так же на основе “дуальных" тождеств

$$
\begin{array}{r}
1-\mathrm{E} z^{\eta_{-}^{0}}=\exp \left\{-\sum_{k=1}^{\infty} \frac{z^{k}}{k} \mathrm{P}\left(S_{k}<0\right)\right\}, \\
1-\mathrm{E}\left(z^{\eta_{+}^{0}} ; \eta_{+}^{0}<\infty\right)=\exp \left\{-\sum \frac{z^{k}}{k} \mathrm{P}\left(S_{k} \geqslant 0\right)\right\} .
\end{array}
$$

Дуальность, представленная в теоремах $2,2 \mathrm{~A}$, сохранится и для всего последующего изложения. Однако ее описание в дальнейшем мы будем опускать ввиду ее очевидности.

Условия, при которых справедливы соотношения (25), (26), могут быть расширены.

Рассмотрим сначала случай, когда $\mathrm{E} \xi^{2}<\infty$. 
Teopema 3.

1. Предположим, что вместо условия $\mathrm{P}(\xi>x) \equiv V(x) \in \mathscr{R}$ (в теореме 2) выполнены следующие условия:

1а) Существует функиия $\widehat{V} \in \mathscr{R}$ такая, что $V(x)$ удовлетворяет соотношениям

$$
V(x) \leqslant \widehat{V}(x), \quad n \widehat{V}^{2}(n)=o(V(|a| n)) ;
$$

1b) $V(h x)<c V(x), c<\infty$, при $x \rightarrow \infty$ июбом фиксированном $h \in(0,1)$;

1c) $V(x+v) \sim V(x)$ при $x \rightarrow \infty,|v|<M \sqrt{x}$ и любом $M<\infty$.

Тогда справедливы соотношения (25), (26).

2. Пусть вместо условия $V(x) \in \mathscr{S e}$ (в теореме 2) выполнены условия

2а) существует функиия $l(x)=x^{\alpha} L(x), \quad \alpha<1 / 2, \quad L-M M \Phi$, удовлетворяющая (6), такая, что

$$
v(x) \equiv-\ln V(x) \geqslant l(x)
$$

2b) $v(x h) / v(x)>\operatorname{ch}^{\gamma}$ npu $x \rightarrow \infty, \gamma \in(\alpha, 1), \quad h<1$;

2c) $v(x+t)-v(x)=o(1)$ при $x \rightarrow \infty, \quad|t| \leqslant M \sqrt{x}$ и любом фиксированном $M<\infty$.

Тогда справедливы соотношения (25), (26).

ДокАЗАТЕЛьство. Здесь остаются в силе все рассуждения доказательства теоремы 2. Надо лишь убедиться, что новые, более широкие, условия по-прежнему влекут выполнение (28) и субэкспоненциальность $\left\{b_{k}\right\}$.

Пусть выполнены условия 1a)-1c). Если использовать метод “срезок" (на уровне $h|a| n)$ при изучении асимптотики $\mathrm{P}\left(S_{n}^{0}>a n\right)($ см., например, [18]), то мы при $h \in(0,1)$ получим в силу $1 \mathrm{a})-1 \mathrm{c})$ соотношение

$$
\mathrm{P}\left(S_{n}^{0}>|a| n\right)=n \mathrm{P}\left(S_{n}^{0}>|a| n, \xi_{n}-a>h|a| n\right)+O\left((n V(h|a| n))^{2}\right),
$$

где при $M$, стремящемся к бесконечности достаточно медленно, в силу неравенства Чебьшева

$$
\begin{aligned}
\mathrm{P}\left(S_{n}^{0}>|a| n, \xi_{n}-a>h|a| n\right)= & \mathrm{P}\left(S_{n-1}^{0}+\xi_{n}-a>|a| n, \xi_{n}-a>h|a| n\right) \\
= & \mathrm{P}\left(S_{n-1}^{0}>(1-h)|a| n\right) V(|a|(h n-1)) \\
& +\int_{-\infty}^{(1-h) a n} \mathrm{P}\left(S_{n-1}^{0} \in d t\right) V(|a|(n-1)-t) \\
= & o(V(n))+\mathrm{E}\left[V\left(|a|(n-1)-S_{n-1}^{0}\right) ; S_{n-1}^{0}<M \sqrt{n}\right] \\
& +\mathrm{E}\left[V\left(|a|(n-1)-S_{n-1}^{0}\right) ; M \sqrt{n} \leqslant S_{n-1}^{0}<h|a| n\right] \\
= & V(|a| n)+o(V(n)) .
\end{aligned}
$$

Это доказьвает, что

$$
\mathrm{P}\left(S_{n}^{0}>|a| n\right) \sim n V(|a| n) .
$$


Субэкспоненциальность $\left\{b_{n}\right\}, b_{n} \sim V(|a| n) / D$, вытекает из соотношений (пусть для простоты $n$ четно)

$$
\sum_{k=0}^{n} b_{k} b_{n-k}=2 \sum_{k=0}^{n / 2-1} b_{k} b_{n-k}+b_{n / 2}^{2}
$$

где

$$
\begin{gathered}
\sum_{k=0}^{n / 2-1}=\sum_{k=0}^{[M \sqrt{n}]}+\sum_{k=[M \sqrt{n}]+1}^{n / 2-1}, \\
\sum_{k=0}^{[M \sqrt{n}]} b_{k} b_{n-k} \sim b_{n}, \quad \sum_{k=[M \sqrt{n}]+1}^{n / 2-1} b_{k} b_{n-k}<c V(|a| n) \sum_{k=[M \sqrt{n}]+1}^{n / 2-1} b_{k}=o\left(b_{n}\right) .
\end{gathered}
$$

Это доказьвает субэкспоненциальность $\left\{b_{n}\right\}$.

Случай, когда вьполнены условия 2a)-2c) рассматривается аналогично с помощью результатов [18], [19].

Теорема 3 доказана.

Совершенно аналогичным образом может быть рассмотрен случай, когда $\mathrm{E} \xi^{2}=\infty$. Пусть для простоты $\alpha \in(1,2)$ в (4) и выполняется

$$
\mathrm{P}(\xi \leqslant-x) \leqslant c V(x) .
$$

ТЕорема 3А. Первое утверждение теоремы 3 в случае (30) полностью сохранится, если в 1с) область $|t|<M \sqrt{x}$ заменить на $|t|<M b(x), \quad b(x)=V^{(-1)}(1 / x)$.

Если условие $\mathrm{P}(\xi \leqslant-x) \leqslant c V(x)$ не вьполнено, то теорема 3 А также остается в силе, но для другой функции $b(x)$ (см. [18]).

ДоКАЗАТЕЛЬСТво ТЕОРЕмЫ 3 А повторяет доказательство теоремы 3. Надо лишь в $(29)$ область уклонений $\left\{S_{n-1}^{0}<M \sqrt{n}\right\}$ заменить на $\left\{S_{n-1}^{0}<M b(n)\right\}$ и воспользоваться оценками в [18].

Случаи $\alpha=1, \alpha=2$ также могут быть рассмотрены.

1.3. Оценки для вероятностей (2). Так как в силу теоремы $1 \mathrm{P}\left(\eta_{+}=n\right)<$ $\mathrm{P}\left(\eta_{-}>n\right)$, то мы остановимся лишь на оценках $\mathrm{P}\left(\eta_{-}>n\right)$.

ТЕОРЕМА 4. Пусть существует функиия $\widehat{V}(x)$, удовлетворяюшая условиям теоремы 2 (или 3), такая, что

$$
V(x)=\mathrm{P}(\xi>x) \leqslant \widehat{V}(x) .
$$

(Другими словами $, \underset{D}{\leqslant} \hat{\xi}, \mathrm{P}(\hat{\xi}>x)=\widehat{V}(x), \hat{\xi}$ удовлетворяет условиям теоремы 2 , если $\mathrm{E} \hat{\xi}=\hat{a}<0$.$) Тогда для любого \varepsilon>0$ и всех достаточно больиих $n$

$$
\mathrm{P}\left(\eta_{-}>n\right) \leqslant \widehat{V}(|a| n(1-\varepsilon)) \mathrm{E} \eta_{-} .
$$

Если $\widehat{V} \in \mathscr{S} e, \alpha \geqslant 1 / 2$, то по-прежснему справедливо соотношение (31) (в случае $\widehat{V} \in \mathscr{S}$ е постоянный мнохитель при $\widehat{V}(|a| n(1-\varepsilon))$ роли не играет, так как малье изменения $\varepsilon$ могут менять $\widehat{V}(|a| n(1-\varepsilon))$ более, чем на постоянный множитель). 
ДокаЗАтЕльство. Утверждение (31) почти очевидно, так как $\eta_{-} \leqslant \underset{D}{\leqslant} \widehat{\eta}_{-}$, где $\widehat{\eta}_{-}$ определяется как $\eta_{-}$, но для случайных величин $\hat{\xi}_{1}, \hat{\xi}_{2}, \ldots$, распределенных как $\hat{\xi}$. Поэтому в силу теоремы 2

$$
\mathrm{P}\left(\eta_{-}>n\right) \leqslant \mathrm{P}\left(\widehat{\eta}_{-}>n\right) \sim \widehat{V}(|\hat{a}| n) \mathrm{E} \widehat{\eta}_{-} .
$$

Но распределение $\hat{\xi}$ можно определить и с помощью соотношений

$$
\mathrm{P}(\hat{\xi}>x)=\left\{\begin{array}{l}
V(x) \text { при } x \leqslant M, \\
\widehat{V}(x) \text { при } x>M,
\end{array}\right.
$$

и выбрать для заданного $\varepsilon>0$ значение $M$ так, чтобы $\hat{a}<a+\varepsilon, \mathrm{E} \widehat{\eta}_{-}<\mathrm{E} \eta_{-}+\varepsilon$. Отсюда и из (32) следует (31).

Докажем теперь второе утверждение. Здесь опять можно использовать приведенные выше рассуждения, но за исключением соотношения вида (28) для сумм $\widehat{S}_{n}=\sum_{i=1}^{n} \hat{\xi}_{i}$. Если $\widehat{V} \in \mathscr{S} e, \alpha \geqslant 1 / 2$, то из результатов [18] следует лишь, что для любого $\varepsilon>0$ и всех достаточно больших $n$

$$
\mathrm{P}\left(\widehat{S}_{n}-\hat{a} n>|\hat{a}| n\right) \leqslant c(n \widehat{V}(|\hat{a}| n))^{1-\varepsilon}=c n^{1-\varepsilon} e^{-l(|\hat{a}| n)(1-\varepsilon)}
$$

(см. следствие 5.1 в [18]). Так как $l(t n) \sim t^{\alpha} l(n)$, то при $\hat{a}<a+\varepsilon$ мы можем правую часть (33) оценить сверху следующим значением (изменив немного $\varepsilon$, если потребуется):

$$
n e^{-l(|a| n(1-\varepsilon))}=n \widehat{V}(|a| n(1-\varepsilon)) .
$$

Поскольку вероятности $\mathrm{P}\left(\eta_{-}>n\right)$ не превышают коэффициентов разложения

$$
\exp \left\{\sum \frac{z^{k}}{k} \mathrm{P}\left(\widehat{S}_{k}>0\right)\right\}
$$

где

$$
\frac{\mathrm{P}\left(\widehat{S}_{k}>0\right)}{k} \leqslant \widehat{V}(|a| k(1-\varepsilon))
$$

при достаточно больших $k$, то рассматриваемые вероятности не превьшают также коэффициентов разложения

$$
\exp \left\{B \sum z^{k} b_{k}\right\}
$$

где

$$
b_{k} \sim \frac{\widehat{V}(|a| k(1-\varepsilon))}{B}, \quad \sum_{k=1}^{\infty} b_{k}=1, \quad B=\sum_{k=1}^{M} \mathrm{P}\left(\widehat{S}_{k}>0\right)+\sum_{k>M} \widehat{V}(|a| k(1-\varepsilon)) .
$$

Так как последовательность $b_{k}$ субэкспоненциальна, полагая $\mathscr{A}(v)=e^{B v}$, мы получим

$$
\mathscr{A}^{\prime}(1)=B e^{B}, \quad \mathrm{P}\left(\eta_{-}>n\right)<\widehat{V}(|a| n(1-\varepsilon)) e^{B}(1+o(1)) .
$$

Поскольку мальм изменением $\varepsilon$ значение $\widehat{V}(|a| n(1-\varepsilon))$ можно уменьшить в $e^{B}$ раз (или другое постоянное число раз), мы получим второе утверждение теоремы.

Теорема доказана. 
СлЕДСТвиЕ 1. Если

$$
E=\mathrm{E}\left(e^{l(\xi)} ; \xi>0\right)<\infty,
$$

l имеет вид (5), то при любом $\varepsilon>0$

$$
E e^{l\left(|a|(1-\varepsilon) \eta_{-}\right)}<\infty, \quad \mathrm{E}\left(e^{l\left(|a|(1-\varepsilon) \eta_{+}\right)} ; \eta_{+}<\infty\right)<\infty .
$$

Это утверждение непосредственно вытекает из теоремы 4 и неравенства Чебьшева

$$
\mathrm{P}(\xi>x)<E e^{-l(x)} .
$$

ЗАмечАние 3. Утверждение (31) в случае $\widehat{V} \in \mathscr{S}$ е можно доказьвать проще, пользуясь грубыми неравенствами (скажем, в случае $\alpha<1 / 2 ;$ см. [18])

$$
\mathrm{P}\left(\eta_{-}>n\right)<\mathrm{P}\left(S_{n}>0\right) \leqslant \mathrm{P}\left(\widehat{S}_{n}>0\right)(1+o(1)) \leqslant n \widehat{V}(|\hat{a}| n) \leqslant \widehat{V}(|a| n(1-\varepsilon)) .
$$

ЗАмечаниЕ 4. В утверждении (31) теоремы 4 можно считать, что $\varepsilon=\varepsilon(n) \rightarrow 0$ при $n \rightarrow \infty$. Скорость сходимости $\varepsilon(n) \rightarrow 0$ можно получить из оценок для $\varepsilon$, содержащихся в [18], и оценок близости $\hat{a}$ и $a$.

1.4. Случай $a=\mathrm{E} \xi<0, x=0$, класс $\mathscr{C}$. Рассмотрим сначала первую из двух возможностей в $(8)$ в случае $V \in \mathscr{C}$. Напомним, что $\varphi(\lambda)=\mathrm{E} e^{\lambda \xi}, \lambda_{0}>0$ - точка, в которой достигается $\varphi=\min \varphi(\lambda), \mu=\sup \{\lambda: \varphi(\lambda)<\infty\}$.

Рассматриваемьй случай $\lambda_{0} \leqslant \mu, \varphi^{\prime}\left(\lambda_{0}\right)=0$ включает в себя два существенно разных подслучая

a) $\lambda_{0}<\mu$,

b) $\lambda_{0}=\mu, \varphi^{\prime}(\mu)=0$.

В случае b) конечность $\varphi(\mu)$ и $\varphi^{\prime}(\mu)$ означает, что

$$
V(t)=e^{-\mu t} V_{0}(t), \quad \int_{0}^{\infty} t V_{0}(t) d t<\infty .
$$

В этом случае нам понадобится

УСЛОВИе (В). Либо $\varphi^{\prime \prime}(\mu)<\infty$, либо $\varphi^{\prime \prime}(\mu)=\infty u$

$$
\widetilde{V}_{0}(t)=\int_{t}^{\infty} V_{0}(u) d u \in \mathscr{R} .
$$

Соотношение (35) означает, что

$$
\widetilde{V}_{0}(t)=t^{-\alpha} \tilde{L}(t)
$$

где $\alpha \in[1,2], \tilde{L}-\mathrm{MM} \Phi$.

Вернемся к общему случаю $\lambda_{0} \leqslant \mu$. Обозначим через $P(B)=\mathrm{P}(\xi \in B)$ распределение $\xi$ и введем в рассмотрение сопряженное к $P$ распределение (преобразование Крамеpa)

$$
P^{*}(d t)=\frac{e^{\lambda_{0} t} P(d t)}{\varphi}
$$


Пусть $\xi_{i}^{*}$ - независимые случайные величины с распределением $P^{*}$. Положим

$$
S_{n}^{*}=\sum_{i=1}^{n} \xi_{i}^{*}
$$

Тогда, очевидно, $\mathrm{E} \xi_{i}^{*}=0$. Если $\lambda_{0}<\mu$, то $\sigma^{2}=\mathrm{E}\left(\xi_{i}^{*}\right)^{2}<\infty$ и $S_{n}^{*} /(\sigma \sqrt{n})$ сходится по распределению к нормальному закону. Если $\lambda_{0}=\mu$, то

$$
V^{*}(t)=\mathrm{P}\left(\xi_{1}^{*}>t\right)=\int_{t}^{\infty} \frac{e^{\lambda_{0} u} P(d u)}{\varphi}=\frac{1}{\varphi} \int_{t}^{\infty}\left(\lambda_{0} V_{0}(u) d u-d V_{0}(u)\right) \sim \frac{\lambda_{0}}{\varphi} \widetilde{V}_{0}(t) .
$$

Это означает, что при вьполнении условия $\lambda_{0}=\mu$ и условия (В) $\xi_{i}^{*}$ принадлежат области притяжения устойчивого закона $F_{\alpha, 1}$ с показателями $(\alpha, 1), \alpha \in[1,2]$ (левый хвост распределения $P^{*}$ экспоненциально убьвает; при $\alpha=2$ предельньй закон $F_{\alpha, 1}$ является нормальным). Распределение $F_{\alpha, 1}$ имеет непрерывную плотность $f$.

Положим

$$
\sigma_{n}= \begin{cases}\sigma \sqrt{n}, & \text { если } \sigma^{2}<\infty, \\ V^{*(-1)}\left(\frac{1}{n}\right), & \text { если } \sigma^{2}=\infty,\end{cases}
$$

где $V^{*(-1)}=\inf \left\{v: V^{*}(v) \leqslant u\right\}-$ функция, обратная к $V^{*}, \widetilde{D}=\sum \mathrm{P}\left(S_{k}>0\right) \varphi^{-k} / k$.

Распределение $P$ мы будем называть нерешетчатым, если его носитель не сосредоточен на множестве $\{b+h k ; k=0, \pm 1, \pm 2, \ldots\}, 0 \leqslant b \leqslant h$, где $h$, не ограничивая общности, можно считать равньм 1. Распределение $P$ мы будем назьвать арифметическим, если $\xi$ целочисленна с шагом решетки, равным 1.

Tеорема 5. Если $P \in \mathscr{C}, \lambda_{0} \leqslant \mu, \varphi^{\prime}\left(\lambda_{0}\right)=0$ и в случае $\lambda_{0}=\mu$ выполнень условия (34), (В), то в нерешетчатом случае

$$
\begin{aligned}
& \mathrm{P}\left(\eta_{-}>n\right) \varphi^{-n} \sim e^{\widetilde{D}} \frac{f(0)}{\lambda_{0} n \sigma_{n}}, \\
& \mathrm{P}\left(\eta_{+}=n\right) \varphi^{-n} \sim e^{-\widetilde{D}} \frac{f(0)}{\lambda_{0} n \sigma_{n}}
\end{aligned}
$$

где $f(0)$ - плотность предельного закона $F_{\alpha, 1}$ в точке 0 .

Если $\xi$ имеет арифметическое распределение, то мнохитель $1 / \lambda_{0}$ в правых частях (39), (40) надо заменить на $e^{-\lambda_{0}} /\left(1-e^{-\lambda_{0}}\right)$.

ДокАЗАТЕльство. Между распределениями $S_{n}$ и $S_{n}^{*}$ существует связь, аналогичная (37):

$$
\mathrm{P}\left(S_{n} \in d t\right)=\varphi^{n} e^{-\lambda_{0} t} \mathrm{P}\left(S_{n}^{*} \in d t\right)
$$

(см., например, соотношение (37) в [2, гл. $8, \S 8]$ ), так что

$$
\mathrm{P}\left(S_{n}>0\right)=\varphi^{n} \int_{0}^{\infty} e^{-\lambda_{0} t} \mathrm{P}\left(S_{n}^{*} \in d t\right)=\varphi^{n} \lambda_{0} \int_{0}^{\infty} e^{-\lambda_{0} u} \mathrm{P}\left(S_{n}^{*} \in(0, u]\right) d u .
$$

Если $\xi_{i}^{*}$ нерешетчаты (носитель $P$ не сосредоточен на множестве $\{b+h k, k=0, \pm 1$, $\pm 2, \ldots\})$, то при выполнении условий $\lambda_{0}<\mu$ или $\lambda_{0}=\mu,(\mathrm{B})$ в силу интегро-локальной теоремы (см. [6, с. 351], [20], [21])

$$
\mathrm{P}\left(S_{n}^{*} \in(0, u]\right)=\mathrm{P}\left(\frac{S_{n}^{*}}{\sigma_{n}} \in\left(0, \frac{u}{\sigma_{n}}\right]\right) \sim \frac{u}{\sigma_{n}} f(0)
$$


равномерно по $u \in\left(0, u_{0}\right)$ при любом $u_{0}>0, \mathrm{P}\left(S_{n}^{*} \in(0, u]\right)<c u / \sigma_{n}$ при всех $u$. Поэтому в силу (41) и теоремы о мажорируемой сходимости

$$
\mathrm{P}\left(S_{n}>0\right) \sim \frac{\lambda_{0} \varphi^{n} f(0)}{\sigma_{n}} \int_{0}^{\infty} u e^{-\lambda_{0} u} d u=\frac{\varphi^{n} f(0)}{\lambda_{0} \sigma_{n}} .
$$

Далее воспользуемся опять факторизационньми тождествами (20), (21), где сделаем замену переменных $s=z \varphi$ и будем следовать доказательству теоремы 2 , но вместо функций $b(z)$ в $(22),(23)$ будем использовать функции

$$
\tilde{b}(s)=b\left(\frac{s}{\varphi}\right)=\sum_{k=1}^{\infty} \tilde{b}_{k} s^{k}
$$

Последовательность

$$
\tilde{b}_{k}=\frac{\mathrm{P}\left(S_{k}>0\right) \varphi^{-k}}{\widetilde{D} k} \sim \frac{f(0)}{\lambda_{0} k \sigma_{k} \widetilde{D}}, \quad \widetilde{D}=\sum \frac{\mathrm{P}\left(S_{k}>0\right) \varphi^{-k}}{k}
$$

является субэкспоненциальной. Поэтому применяя вновь результаты [15], получим

$$
\mathrm{P}\left(\eta_{-}>n\right) \varphi^{-n} \sim e^{\widetilde{D}} \frac{f(0)}{\lambda_{0} k \sigma_{k} \widetilde{D}} .
$$

В решетчатом случае, когда $\xi$ целочисленна с шагом решетки 1 , следует воспользоваться локальной теоремой

$$
\sum_{k}\left|\mathrm{P}\left(S_{n}^{*}=k\right)-\frac{f\left(k / \sigma_{n}\right)}{\sigma_{n}}\right| \rightarrow 0
$$

при $n \rightarrow \infty$ (см. [22, теорема 4.2.2]), из которой следует, что

$$
\mathrm{P}\left(S_{n}>0\right)=\varphi^{n} \sum_{k=1}^{\infty} e^{-\lambda_{0} k} \mathrm{P}\left(S_{n}^{*}=k\right) \sim \frac{\varphi^{n} e^{-\lambda_{0}} f(0)}{\left(1-e^{-\lambda_{0}}\right) \sigma_{n}} .
$$

Доказательство второго утверждения теоремы происходит совершенно аналогично.

Теорема доказана.

Отметим, что в случаях $\lambda_{0}<\mu$ или $\lambda_{0}=\mu, \varphi^{\prime \prime}(\mu)<\infty$ в (39), (40) вьполняется $f(0)=1 / \sqrt{2 \pi}, \sigma_{n}=\sigma \sqrt{n}, \sigma^{2}=\varphi^{\prime \prime}\left(\lambda_{0}\right) / \varphi$.

Заметим также, что решетчатьй неарифметический случай, когда $\xi$ принимает значения $b+h k ; k=0, \pm 1, \ldots, 0<|b|<h$, также может быть рассмотрен, однако в этом случае коэффициент при $1 /\left(n \sigma_{n}\right)$ в правых частях $(39),(40)$ будет зависеть от $n$, колеблясь в фиксированных пределах.

Рассмотрим теперь в (8) вторую возможность $\lambda_{0}=\mu, \varphi^{\prime}(\mu)<0$. Здесь по-прежнему справедливо (34). Предположим, что $V_{0} \in \mathscr{R}$, т.е.

$$
V_{0}(t)=t^{-\alpha-1} L_{0}(t), \quad \alpha>1,
$$

где $L_{0}-\mathrm{MM} \Phi$. 
Tеорема 6. Если $P \in \mathscr{C}, \lambda_{0}=\mu, \varphi^{\prime}(\mu)<0$, выполнено (34), (43), то

$$
\mathrm{P}\left(\eta_{-}>n\right) \varphi^{-n} \sim e^{\tilde{D}} \frac{V\left(a_{+} n\right)}{\varphi}, \quad \mathrm{P}\left(\eta_{+}=n\right) \varphi^{-n} \sim e^{-\tilde{D}} \frac{V\left(a_{+} n\right)}{\varphi},
$$

əде $a_{+}=-\mathrm{E} \xi^{*}=-\frac{\varphi^{\prime}(\mu)}{\varphi}>0$.

ДокАЗАТЕльство. В условиях теоремы 6 по-прежнему справедливо равенство (41) для $\mathrm{P}\left(S_{n}>0\right)$, где $\lambda_{0}$ можно заменить на $\mu$, так что задача опять сводится к отысканию асимптотики $\mathrm{P}\left(S_{n}^{*} \in(0, u]\right)=\mathrm{P}\left(S_{n}^{*}+a_{+} n \in\left(a_{+} n, a_{+} n+u\right]\right)$. Имеем

$$
\mathrm{P}\left(\xi_{1}^{*} \in(t, t+u]\right)=\int_{t}^{t+u} \frac{e^{\mu v} P(d v)}{\varphi}=\int_{t}^{t+u} \frac{\mu V_{0}(u)}{\varphi} d v-\int_{t}^{t+u} \frac{d V_{0}(v)}{\varphi} \sim \frac{\mu u V_{0}(t)}{\varphi} .
$$

Это означает, что при каждом фиксированном $u$ выполнены условия теорем 2.1,3.1 в [23] и для уклонений порядка $n$ (для центрированных сумм $S_{n}^{*}+a_{+} n$ ) имеем

$$
\mathrm{P}\left(S_{n}^{*} \in(0, u]\right) \sim \frac{\mu u n V_{0}\left(a_{+} n\right)}{\varphi \alpha} .
$$

Отсюда в силу (41), как и прежде, находим

$$
\mathrm{P}\left(S_{n}>0\right) \varphi^{-n} \sim \mu \int_{0}^{\infty} e^{-\mu u} u d u \cdot \frac{\mu n V_{0}\left(a_{+} n\right)}{\varphi \alpha}=\frac{n V_{0}\left(a_{+} n\right)}{\varphi \alpha}
$$

Таким образом, здесь в обозначениях (42)

$$
\tilde{b}_{k}=\frac{\mathrm{P}\left(S_{k}>0\right) \varphi^{-k}}{\widetilde{D} k} \sim \frac{V_{0}\left(a_{+} n\right)}{\varphi \alpha \widetilde{D}}
$$

Последовательность $\tilde{b}_{k}$, как и в теореме 5 , также будет субэкспоненциальной и, следовательно, все остальные рассуждения предыдущего доказательства остаются в силе.

Теорема доказана.

По-видимому, не представляет большого труда распространить утверждения теоремы 6 на случай $V_{0} \in \mathscr{S} e, \alpha<1 / 2$.

Отметим, что предположение (43), как показьвает соотношение (44), соответствует нерешетчатому случаю. Решетчатьй случай может быть рассмотрен совершенно аналогично, но он потребует изменения формы условия (43). Отметим также, что локальные теоремы 2.1, 3.1 в [23] для решетчатого случая ранее установлены в [24]. 


\section{СПИСОК ЦИТИРОВАННОЙ ЛИТЕРАТУРЫ}

[1] Феллер В. Введение в теорию вероятностей и ее приложения. Т. 2. М.: Мир, 1984.

[2] Боровков А. А. Теория вероятностей. М.: Наука, 1986.

[3] Боровков А. А. Вероятностные процессы в теории массового обслуживания. М.: Наука, 1972.

[4] Боровков А. А. Предельные теоремы о распределении максимума сумм ограниченных решетчатых случайных величин. I, II // Теория вероятн. и ее прим. 1960. Т. 5. № 2. С. 137-171; 1960. T. 5. № 4. C. 377-392.

[5] Emery D. J. Limiting behaviour of the distribution of the maxima of partial sums of certain random walks // J. Appl. Probab. 1972. V. 9. P. 572-579.

[6] Bingham N. H., Goldie C. M., Teugels J. L. Regular Variation. Cambridge: Cambridge University Press, 1987.

[7] Doney R. A. On the asymptotic behaviour of first passage times for transient random walk // Probability Theory and Related Fields. 1989. V. 18. P. 239-246.

[8] Bertoin J., Doney R. A. Some asymptotic results for transient random walks // Adv. Appl. Probab. 1996. V. 28. P. 207-227.

[9] Могульский А. А., Рогозин Б. А. Случайные блуждания в положительном квадранте I-III // Матем. труды. 1999. Т. 2. № 2. С. 57-97; 2000. Т. 3. № 1. С. 48-118; 2001. Т. 4. №1. C. $1-25$.

[10] Heyde C. C. Asymptotic renewal results for a natural generalization of classical renewal theory // J. Roy. Statist. Soc. Ser. B. 1967. V. 29. P. 141-150.

[11] Janson S. Moments for first-passage and last-exit times. The minimum, and related quantities for random walks with positive drift // Adv. Appl. Probab. 1986. V. 18. P. 865-879.

[12] Gut A. Stopped random walks // Limit Theorems and Applications. Springer-Verlag, 1988.

[13] Kesten H., Maller R. A. Two renewal theorems for general random walks tending to infinity // Probability Theory and Related Fields. 1996. V. 106. № 1. P. 1-38.

[14] Боровков А. А. Эргодичность и устойчивость случайных процессов. М.: Эдиториал УРСС; Новосибирск: Изд-во ИМ СО РАН, 1999.

[15] Chover J., Ney P., Wainger S. Function of probability measures // J. Analyse Math. 1973. V. 26. P. 255-302.

[16] Asmussen S. Subexponential asymptotics for stochastic processes: extremal behaviour, stationary distributions and first passage probabilities // The Annals of Appl. Probability. 1998. V. 8. № 2. P. 354-374.

[17] Нагаев С. В. Об асимптотическом поведении вероятностей односторонних болшших уклонений // Теория вероятн. и ее примен. 1981. Т. 26. № 2. С. 369-372.

[18] Боровков А. А. Оценки для распределения сумм и максимумов сумм случайных величин при невыполнении условия Крамера // Сиб. матем. ж. 2000. Т. 5. № 5. С. 997-1038.

[19] Боровков А. А. Вероятности больших уклонений для случайных блужданий с семиэкспоненциальньми распределениями // Сиб. матем. ж. 2000. Т. 41. №6. С. 1290-1324.

[20] Stone C. On local and ratio limit theorems // Proc. Fifth Berkeley Symp. Math. Stat. Prob. / ed. J. Neyman. V. 2. №2, 1967. P. 217-224.

[21] Feller W. On regular variation and local limit theorems // Proc. Fifth Berkeley Symp. Math. Stat. Prob. / ed. J. Neyman. V. 2. № 1, 1967. P. 373-388.

[22] Ибрагимов И. А., Линник Ю. В. Независимые и стационарно связанные величины. М.: Наука, 1965.

[23] Боровков А. А. Интегро-локальные теоремы о больших уклонениях сумм случайных векторов. Регулярные распределения // Сиб. матем. ж. 2002. Т. 43. № 3. С. 508-525.

[24] Doney R. A. A large deviation local limit theorem // Math. Proc. Cambridge Phil. Soc. 1989. V. 105. P. 575-577.

Институт математики им. С. Л. Соболева СО РАН, г. Новосибирск 